\title{
Generation of a fusion protein containing the two functional coiled-coil domain of $t$ - SNARE, SNAP-23 and a transmembrane domain for mast cell
}

\author{
D. M. Agase* \\ Department of Zoology, Govt. J.S.T.P.G. College, Balaghat (M.P.), India \\ S. B. Zade \\ Department of Zoology, R.T.M. Nagpur University, Nagpur (MS), India \\ T.S. Kothe \\ Department of Zoology, Govt. K.N.G. College, Balaghat (M.P.), India \\ *Corresponding author. Email: sbt.durgesh@gmail.com
}

\section{Article Info}

https://doi.org/10.31018/

jans.v12i4.2439

Received: November 3, 2020

Revised: December 10, 2020

Accepted: December 13, 2020

\section{How to Cite}

Agase D. M. et al. (2020). Generation of a fusion protein containing the two functional coiled-coil domain of t- SNARE, SNAP-23 and a transmembrane domain for mast cell. Journal of Applied and Natural Science, 12(4):670 - 674. https:// doi.org/10.31018/jans.v12i4.2439

\begin{abstract}
SNAREs (Soluble $\mathrm{N}$-Ethylmaleimide-Sensitive Fusion Protein Attachment Protein Receptor) are a class of membrane proteins that mediate membrane-membrane fusion in eukaryotic cells. SNAP-23 is a t-SNARE which is a component of cellular machinery is required for membrane fusion. SNAP-23 lacks transmembrane domain. Cysteines in the linker region of SNAP-23 are involved in targeting of SNAP-23 to the membrane. In the present work, a portion of MDR3 gene (MDR3 1-145) and CLP24 $\left(\mathrm{CLP}_{134-195}\right)$ was subcloned into a plasmid encoding EGFP-SNAP-23 Cys ${ }^{-}$mutant for the generation of a fusion protein containing the two functional coiled-coil domain of t-SNARE, SNAP 23 and a transmembrane domain of MDR3 gene and CLP24 for mast cell. This fusion protein will be important to study the membrane targeting and raft association of the chimeric SNAP23 protein, which plays an important role in mast cell exocytosis in the mammalian system. A novel bioinformatics approach has been applied to identify the specific transmembrane domain. This novel approach can be used to construct other fusion proteins.
\end{abstract}

Keywords: EGFP, Exocytosis, Fusion Protein, Mast cell, MDR3, SNAREs, SNAP23

\section{INTRODUCTION}

Biological membrane fusion is the central process in all living organisms. It contributes to a variety of biological processes including viral infection, cell fertilization, as well as intracellular transport, and neurotransmitter release. In particular, the various membrane-enclosed compartments in eukaryotic cells need to exchange their contents and communicate across membranes (Han et al., 2017). Members of the SNARE (Soluble $N$ Ethylmaleimide-Sensitive Fusion Protein Attachment Protein Receptor) superfamily are required for intracellular membrane fusion events in eukaryotes (Chen et al., 2001, Buxton et al., 2003, Blumenthal et al., 2003). SNARE proteins are classified into two classes based on their membrane localization. SNARE proteins associated with the vesicle's membrane are termed vesicleSNAREs (v-SNAREs) and those on the target plasma membrane are called target-SNAREs (t-SNAREs) (Ramakrishnan et al., 2012). SNAP-23 (Synaptosomal associated protein of $23 \mathrm{kDa}$ ) is a ubiquitously expressed t-SNARE protein that belongs to the SNAP-25 family (Jahn and Scheller, 2006). Like SNAP25, SNAP -23 is present in many types of cells at the plasma membrane domain, with a little intracellular localization, and mediates exocytosis of secretory vesicles (Sakuraia et al., 2012). Structurally, SNAP-23 lacks a transmembrane domain but contains two SNARE motifs (coiled-coiled domains) connected by a linker region containing five cysteines (Hepp et al., 2005). This cysteine-rich linker region plays a crucial role in raft localization. But the molecular mechanism elucidating the role of these cysteines in targeting of SNAP-23 is not well known. The present work was carried out with the aim to construct a SNAP-23 fusion protein in which the cysteine-rich linker would be replaced by a transmembrane domain of some non-raft protein. This construct would help us in investigating the role of palmitoylation of the cysteines in membranemembrane microdomain association of SNAP-23 and, further in membrane fusion events during exocytosis.

\section{MATERIALS AND METHODS}

Human Lung Carcinoma cell line A549 was a gift from 
Dr.Rajiv K. Saxena, School of life Sciences Jawaharlal Nehru University, New Delhi, and Human liver carcinoma cell line HepG2 was a gift from Dr. Rakesh K. Tyagi, Special Centre for Molecular Medicine, Jawaharlal Nehru University, New Delhi. All cell lines were grown adherent as a monolayer and cultured in Dulbecco's MEM (Sigma) with 10\% FBS in a humidified atmosphere containing $5 \% \mathrm{CO}_{2}$ at $37^{\circ} \mathrm{C}$, according to standard mammalian tissue culture protocols and sterile technique. The doubling time of A549 cells is 40 hrs. and the doubling time of HepG2 and LS180 cells is approximately $27-30 \mathrm{hrs}$. Guidelines of human ethics committee were followed for these experiments. pEGFP- hSNAP-23 Cys ${ }^{-}$plasmid was a gift from Dr. Paul. A. Roche, NIH, USA. The primers were designed for MDR3 (1-145 amino acid residues), and CLP24 (134-195 amino acid residue) using the guidelines for primer designing and were then tested for various parameters like length of primer, GC content, Tm, selfcomplementarities, hairpin formation, etc., using on line tools. Primers were custom synthesized by Sigma Aldrich at $25 \mathrm{nmol}$ scale and purified by desalting. The lyophilized primers were reconstituted in 1x TE (10 mM Tris, $1 \mathrm{mEDTA}$ to prepare $100 \mu \mathrm{m}$ stocks. From these stocks $10 \mu \mathrm{m}$ working solution was made.

A bioinformatic approach was followed for identification of a potential transmembrane protein that has multispanning domains, and not having the targeting sequence for lipid rafts (Fig.1). The search was performed by using different databases: The European Bioinformatics Institute databases- UniProt, SwissProt, the ExPASy (Expert Protein Analysis System) proteomics server. This broad search yielded a large

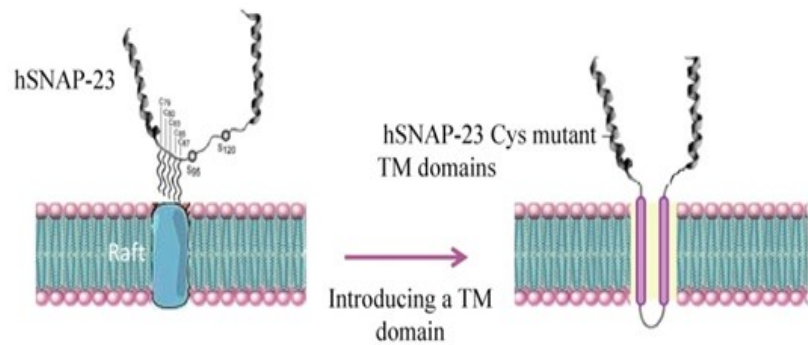

Fig. 1. Schematic representation of SNAP-23 wild type protein and SNAP-23 fusion protein in which the cysteine-rich linker was to be replaced by a transmembrane domain.

number of potential target proteins. These proteins were studied in detail for their membrane topology, functions, sequence analysis, post-translational modifications, tissue-specific expression by using TMMOD, GPI-SOM, NMT online analysis tools and the search was first narrowed down to a few potential protein candidates and then narrowed down to a few transmembrane domains from these proteins (Kahsay et al., 2005, Pierleoni and Martelli, 2008).

A cloning strategy (Fig.2) was followed to construct trans-membrane SNAP-23 fusion protein that does not go to the lipid rafts. RNA was isolated from A549 and HepG2 cell lines by Trizol reagent method (Chomczynski et al., 1987). RNA recoveries were estimated by using Nanodrop ND3000 Spectrophotometer. 1.2\% Formaldehyde Agarose Gel electrophoresis was used to visualized RNA. Gradient PCRs were performed for $\mathrm{CLP}_{134-195}$ and MDR3 1-145 $_{1}$ gene amplification using sequence-specific primers incorporating the
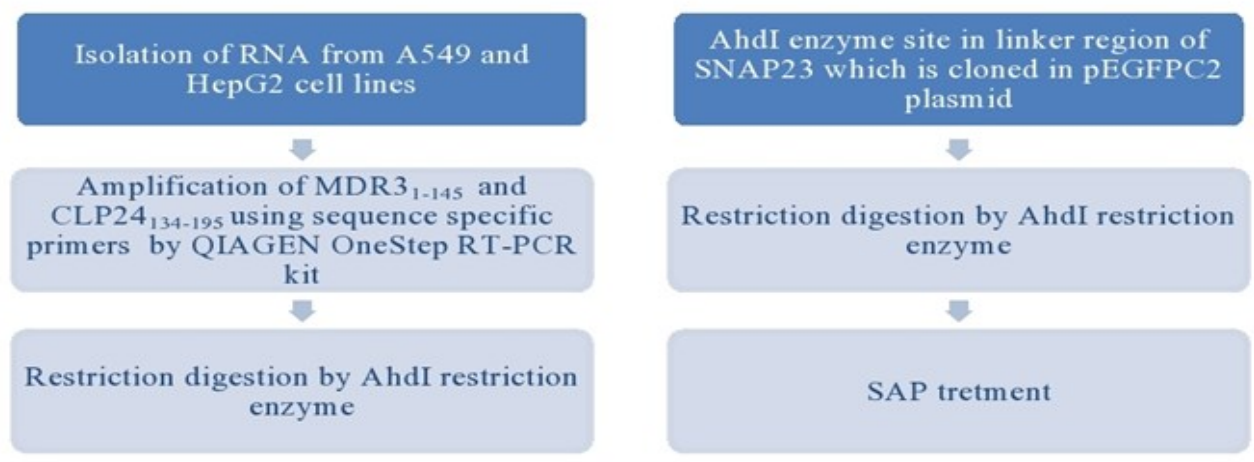

I

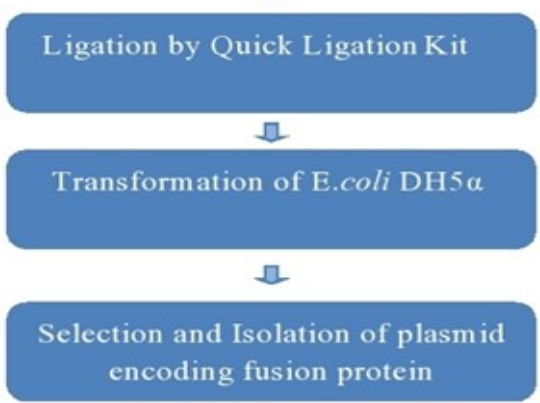

Fig. 2. Cloning strategy to construct trans-membrane, non-raft SNAP-23 fusion protein for mast cell. 
Ahdl site present in the linker region of SNAP-23 by QIAGEN OneStep RT-PCR kit and RNA of A549 and HepG2 as a template. The amplified products were digested with Ahdl and ligated into the same Ahdl site present in plasmid encoding EGFP-SNAP-23 Cysmutant. Ligation was carried out by Quick Ligation ${ }^{\text {TM }}$ Kit (from NEB). It enables ligation of cohesive end or blunt end DNA fragments in 5 minutes at room temperature. The ligated product was transformed into $E$ coli $D H 5 \alpha$ competent cells and selected using an antibiotic selection marker. Miniprep of all the transformed colonies was done and isolated plasmid was checked by digestion with same Ahdl restriction enzyme to see the in-

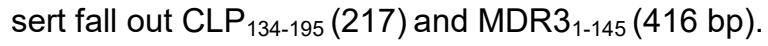

\section{RESULTS AND DISCUSSION}

In the present study, 50 proteins were found to be associated with the terms "human OR rat AND multi AND membrane-spanning NOT lipid-rafts" in InterPro, UniProt, ExPASy and NCBI protein databases. Among them, 10 proteins were common. These proteins were membrane-spanning which belonged to human and did not show any association with membrane raft. The common proteins were selected on the basis of their complete structural details; individual work on membrane targeting domains does not participate in any signalling function. Finally, this In silico search yielded two membrane proteins that were found to be potential candidates for use in generating fusion protein: Human membrane glycoprotein P (MDR3) and Transmembrane Protein 204 (CLP24).

After Functional analysis, Membrane Topology and Post-translational Modifications study, the polypeptide sequence MDR3 $_{1-145}$ (AA,1-145) and CLP24 ${ }_{134-195}$ (AA 134-195) were selected. These polypeptide segment of MDR3 and CLP23 protein fulfilled the necessary criteria to be used for the generation of the fusion protein. In silico gene expression profile shown that human MDR3 gene is expressed at a higher level in hepatic tissues (liver). HepG2 cell line is a hepatic carcinoma cell line that expresses MDR3 gene at a higher level and used for isolation of RNA and amplification of the desirable gene sequence by Polymerase chain reaction (PCR). Similarly, In silico gene expression profile showed that human CLP24 gene is expressed at the higher level in the lungs. A549 cell line is a human alveolar basal epithelial carcinoma cell line that expresses CLP24 gene at higher level and is a potential candidate for isolation of RNA for amplification of desirable gene sequence by PCR.

Isolated RNA was of good quality and amount of RNA isolated was higher from A549 cells as compared to HepG2 cells. RNA gel (1.2\% Formaldehyde Agarose) showed distinct bands of $28 \mathrm{~s}$ and $18 \mathrm{~s}$ for RNA samples along with 7 bands of ssRNA ladder (Fig. 3). The amplified DNA fragment for CLP $_{134-195}$ was seen at $50.1^{\circ} \mathrm{C}, 50.6^{\circ} \mathrm{C}, 52.4^{\circ} \mathrm{C}$ and $53.2^{\circ} \mathrm{C}$. By band quantization $50.6^{\circ} \mathrm{C}$ was chosen to be the appropriate anneal- ing temperature. The length of the amplified DNA was $217 \mathrm{bp}$ which was observed slightly above the corresponding 200 bp band of a ladder (Fig. 4). Similarly, the amplified DNA fragment for MDR3 1-145 was seen at $43.8^{\circ} \mathrm{C}$ (Fig. 5). In order to confirm that the right plasmid DNA had been amplified by mini preparation, the plasmid samples were linearized by restriction digestion and then ran on the gel. The restriction digestion of the plasmid pEGFP and pEGFPhSNAP23-Cys ' by Hindlll restriction enzyme showed that the size of the wild type plasmid (pEGFP) is 4.7 $\mathrm{kbp}$ and that of Cysteine mutant plasmid pEGFPhSNAP23-Cys ${ }^{-}$) is $5.5 \mathrm{kbp}$ (Fig. 6). The digested plas-

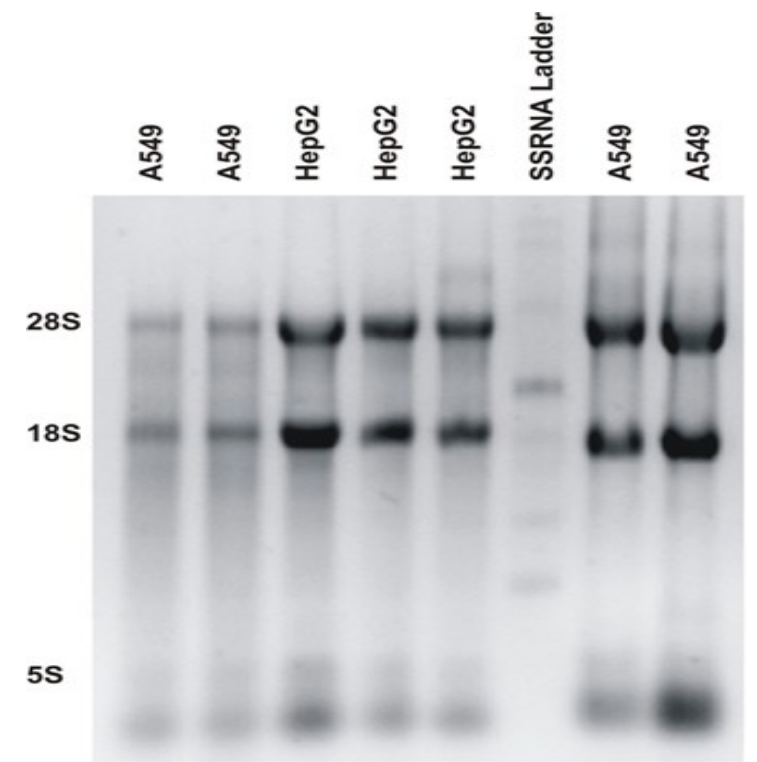

Fig. 3. Formaldehyde Agarose $\mathrm{Gel}$ Electrophoresis of RNA extracted from HepG2 and A549 cell lines (1.2\% Formaldehyde Agarose). RNA gel showing three distinct bands of 28s $18 \mathrm{~s}$ and $5 \mathrm{~s}$ for RNA samples along with ssRNA ladder.

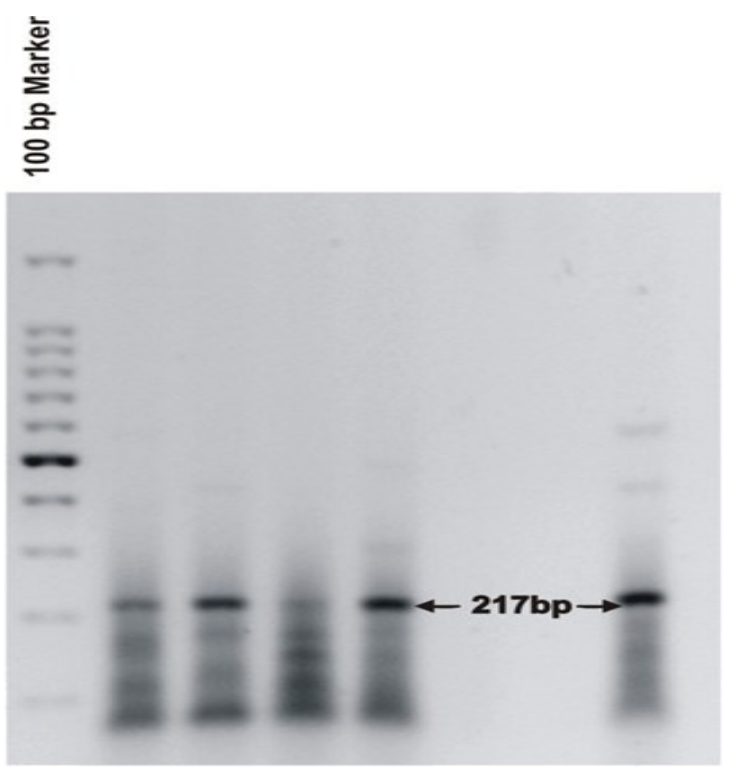

Fig. 4. Agarose Gel electrophoresis (1.8\%), Temperature Gradient showing 217 bp bands for CLP $134-195$. 


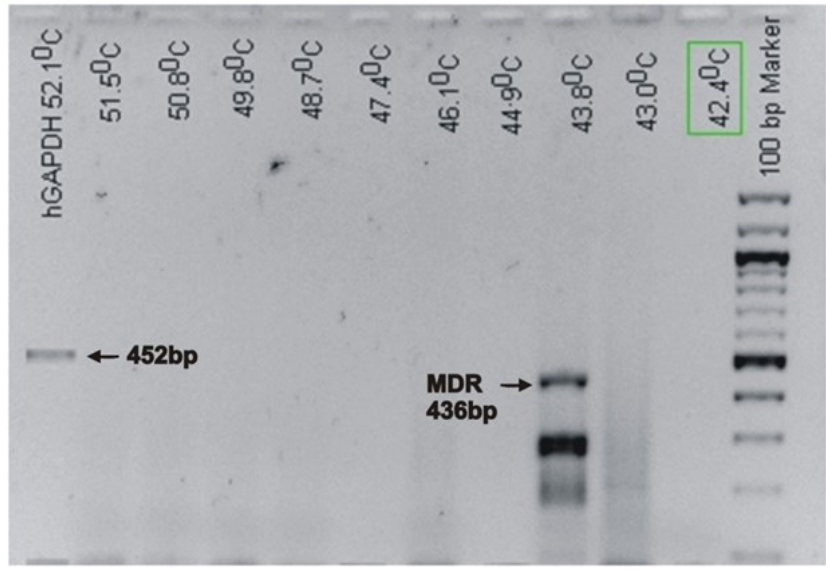

Fig. 5. Agarose Gel electrophoresis (1.8\%), Temperature Gradient showing $436 \mathrm{bp}$ band for MDR3 ${ }_{1-145}$.

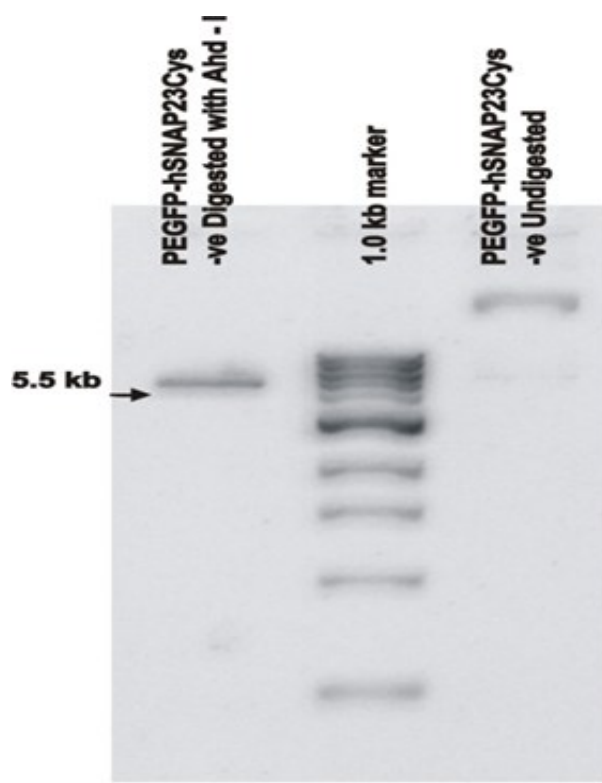

Fig. 6: BamHI digested transformed plasmids $p E G F P$ and pEGFPS23-Cys".

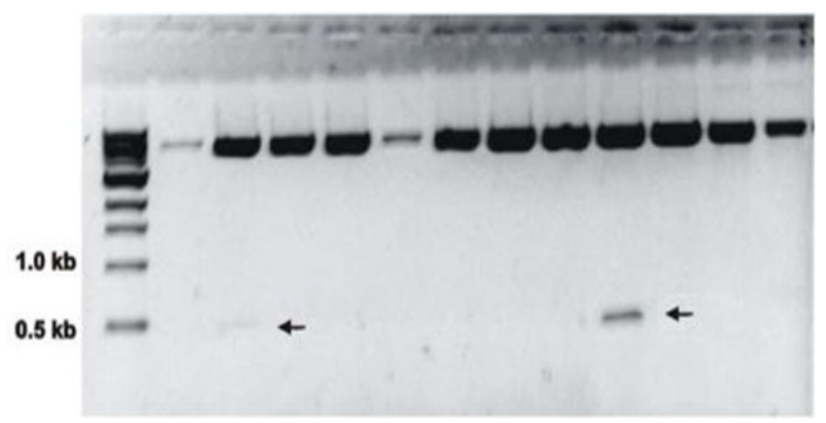

Fig. 8. Transformed, isolated plasmid checked by restriction digestion with Ahdl showing two inserts fall out of MDR3 $1-145$ (416 bp).

mid was linear and hence showed a single band as compared to a native undigested plasmid, which shows multiple bands. Restriction digestion of pEGFPhSNAP23-Cys plasmid with Ahdl, which cut the plasmid in the linker region of SNAP23 showed a linearized single band of $5.5 \mathrm{kbp}$ size. Restriction digestion of insert (MDR3 1-145 and CLP ${ }_{134-195}$ ) with same restriction Enzyme Ahdl showed a slight difference between digested and undigested band $2.0 \%$ agarose gel (Fig. 7). After ligation and transfection, three colonies were found positive, which shows the MDR3 $1-145$ insert fallout of $416 \mathrm{bp}$ and CLP $_{134-195}$ insert fallout of 217 bp (Fig. 8-9).

A longstanding goal in protein engineering is to identify specific sequence changes that endow proteins with desired functional properties. However, constructions of many mutant SNAP-23 fusion proteins have been reported (Bark et al., 1994, Cabaniols et al., 1999, Suh et al., 2011, Klein et al., 2016, Suzuki et al., 2008). As opposed to traditional rational and random protein engineering techniques, we have applied a novel bioinformatic approach to identify specific transmembrane

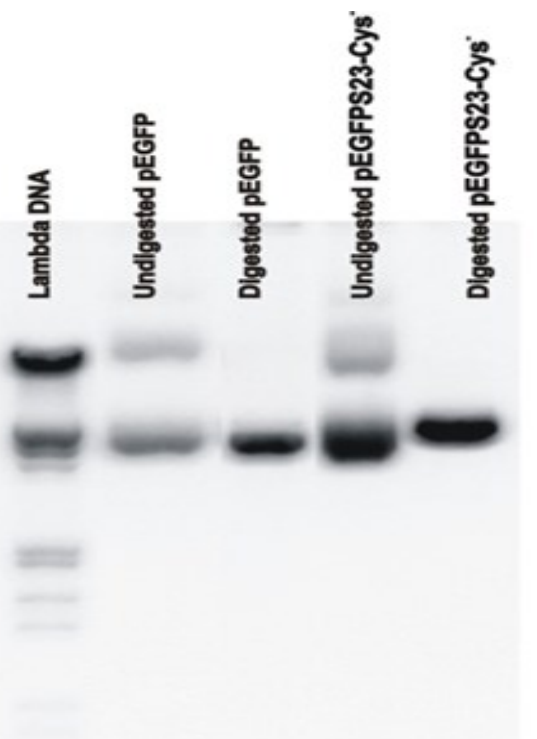

Fig 7: Ahdl digested transformed pEGFP-hSNAP23-Cys plasmid.

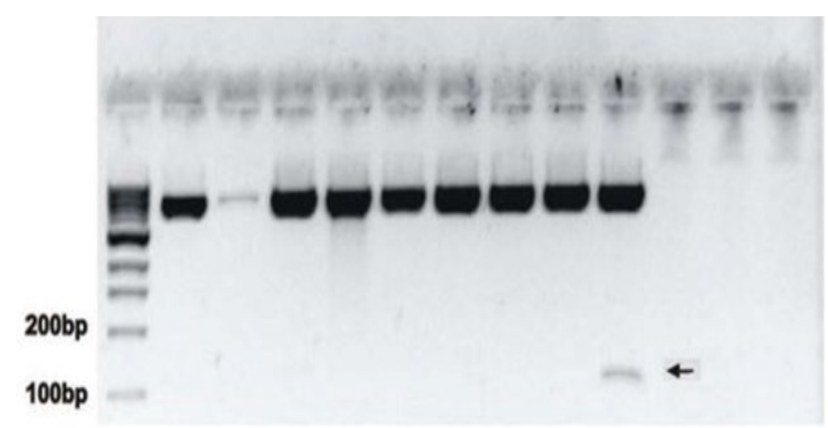

Fig. 9. Showing one insert fall out of CLP ${ }_{134-195}$ (217bp).

domain changes that influenced key topological and functional properties of SNAP-23 protein. The pEGFP plasmid encoding full-length wild-type SNAP-23 and its phosphomutants have been reported by Naskar and Puri, (2017). SNAP-23 Cys mutant, devoid of all five cysteines, and SNAP-23 P119A (proline to alanine) 
mutant, that likely interferes with palmitoylation of SNAP-23 as reported by Agarwal et al. (2019). In the present work, a portion of MDR3 gene (MDR3 ${ }_{1-1} 145$ ) and CLP24 (CLP $134-195)$ was separately subcloned into a plasmid encoding EGFP-SNAP-23 Cys mutant for the generation of a fusion protein containing the two functional coiled-coil domain of t-SNARE, SNAP 23 and a transmembrane domain of MDR3 gene and CLP24 gene. This fusion protein will be important to study the membrane targeting and raft association of the chimeric SNAP23 protein, which could play an important role in mast cell exocytosis in the mammalian system.

\section{Conclusion}

We have successfully cloned a GFP fusion protein containing the two functional coiled-coil domain of $t$ SNARE, SNAP 23 and a transmembrane domain (MDR3 gene and CLP24 gene). This fusion protein will be important to study the membrane targeting and raft association of SNAP-23. A novel bioinformatics approach has been applied to identify the specific transmembrane domain. This novel approach can be used to construct other fusion proteins.

\section{ACKNOWLEDGEMENTS}

We thank Dr Paul A. Roche (NIH, USA) for the kind gift of pEGFP- hSNAP-23 Cys ${ }^{-}$plasmid. We also thank Dr.Rajiv K. Saxena School of life Sciences Jawaharlal Nehru University, New Delhi, Dr. Rakesh K. Tyagi, Special Centre for Molecular Medicine, Jawaharlal Nehru University, New Delhi. and Dr. Niti Puri School of life Sciences Jawaharlal Nehru University, New Delhi for the kind gift of cell lines and their guidance.

\section{Conflict of interest}

There is no conflict of interest regarding the publication of this article.

\section{REFERENCES}

1. Agarwal, V., Naskar, P., Agasti S., Khurana, G.P., Vishwakarma, P., Lynn, A.M., Roche, P.A, and Puri, N., (2019). The cysteine-rich domain of synaptosomal-associated protein of $23 \mathrm{kDa}(\mathrm{SNAP}-23)$ regulates its membrane association and regulated exocytosis from mast cells, Biochimica et Biophysica Acta (BBA) - Molecular Cell Research, 1866:1618-1633. https://doi.org/1 0.1016/j.bbamcr.2019.06.015.

2. Bark I.C., and Wilson, M.C., (1994). Human cDNA clones encoding two different isoforms of the nerve terminal protein SNAP-25, Gene, 139: 291-292. https:// doi.org/10.1016/0378-1119(94)90773-0.

3. Buxton, P., Zhang, X. M., Walsh, B., Sriratana, A., Schenberg, I., Manickam, E. and Rowe, T. (2003) Identification and characterization of Snapin as a ubiquitously expressed SNARE-binding protein that interacts with SNAP23 in non-neuronal cells, Biochem. J, 375:433-440. https://doi.org/10.1042/bj20030427.

4. Cabaniols, J., Ravichandran, V., and Roche P.A. (1999). Phosphorylation of SNAP-23 by the novel kinase SNAK regulates t-SNARE complex, Mol. Biol. Cell, 10:40334041. doi: 10.1242/bio.025791.

5. Chen YA., and Scheller, R.H. (2001). SNARE-mediated membrane fusion, Nature Reviews Molecular Cell Biology, 2: 98-106. https://doi.org/10.1038/35052017.

6. Chomczynski, P., and Sacchi, N. (1987) Single-step method of RNA isolation by acid thiocyanate-phenol-chloroform extraction, Analytical Biochemistry, 62:156-9. https:// doi.org/10.1016/0003-2697(87)90021-2.

7. Han, J., Pluhackova, K. and Bockmann, R. A. (2017). The multifaceted role of SNARE proteins in membrane fusion, Frontiers in Physiology, 8: 1-17. https://doi.org/10.3389/ fphys.2017.00005.

8. Hepp, R., Puri N., Hohenstein, A.C., Crawford, Whiteheart, G.L., and Roche P.A., (2005). Phosphorylation of SNAP-23 regulates exocytosis from mast cells, J. Biol. Chem, 280:6610-6620. DOI 10.1074/jbc.M412126200.

9. Jahn, R., and Scheller, R. (2006). SNAREs - engines for membrane fusion, Nature Reviews Molecular Cell Biology, 7:631-643. https://doi.org/10.1038/nrm2002.

10.Kahsay, R.Y., Guang, G., and Liao, L. (2005). An improved hidden Markov model for transmembrane protein detection and topology prediction and its applications to complete genomes, Bioinformatics, 21:1853-8. https:// doi.org/10.1093/bioinformatics/bti303.

11.Klein, O., Roded, A., Zur N., Puri, N., Pasternak, O., Hirschberg, K., Hammel, I., Roche, P.A., Yatsu, A., Fukuda M., Galli S.J., Eisenberg R.S., (2017). Rab5 is critical for SNAP23 regulated granule-granule fusion during compound exocytosis, Scientific Reporter, 7 :1-17. DOI:10.1038/s41598-017-15047-8.

12.Naskar, P., and Puri, N., (2017). Phosphorylation of SNAP-23 regulates its dynamic membrane association during mast cell exocytosis, Biology Open, 6 :1257-1269. doi: 10.1242/bio.025791.

13.Pierleoni, A., and Martelli, P.L., (2008). PredGPI: a GPIanchor predictor. BMC Bioinformatics, 9 :1-11. https:// doi.org/10.1186/1471-2105-9-392.

14. Blumenthal, R., Michael, J.C., Stewart, R.D., and Richard, M.E. (2003). Membrane Fusion, Chem. Rev, 103:53-69. https://doi.org/10.1021/cr000036+

15.Ramakrishnan, N.A, Drescher, M. J. and Drescher, D.G. (2012). The SNARE complex in neuronal and sensory cells, Mol Cell Neurosci, 50:58-69. https://doi.org/10.1016/ j.mcn.2012.03.009.

16.Sakuraia, C., Hashimotoa, H., Nakanishia, H., Araia, S., Wadab, Y., Wadac, G., Wadaa, I., and Hatsuzawaa, K. (20012). SNAP-23 regulates phagosome formation and maturation in macrophages, Molecular Biology of the Cell, 23: 4849-4863. https://doi.org/10.1091/mbc.e12-01-0069.

17.Suh, Y.H., Yoshimoto F.A, Weih K.A., Tessarollo L, Roche K.W, Mackem S., Roche P.A. (2001). Deletion of SNAP-23 results in pre-implantation embryonic lethality in mice, PLoS One, 6: e18444. https://doi.org/10.1371/ journal.pone.0018444.

18.Suzuki K., and Verma I.M., (2008). Phosphorylation of SNAP-23 by lkB Kinase 2 Regulates Mast Cell Degranulation, Cell, 134: 485-495. https://doi.org/10.1016/ j.cell.2008.05.050. 